\title{
Decoherence of the Radiation from an Accelerated Quantum Source
}

\author{
Daiqin $\mathrm{Su}^{*}$ and Timothy C. Ralph \\ Centre for Quantum Computation and Communication Technology, School of Mathematics and Physics, \\ The University of Queensland, St. Lucia, Queensland, 4072, Australia
}

(Received 21 December 2017; revised manuscript received 9 October 2018; published 11 January 2019)

\begin{abstract}
Decoherence is the process via which quantum superposition states are reduced to classical mixtures. Decoherence has been predicted for relativistically accelerated quantum systems; however, examples to date have involved restricting the detected field modes to particular regions of space-time. If the global state over all space-time is measured, then unitarity returns and the decoherence is removed. Here, we study a decoherence effect associated with accelerated systems that cannot be explained in this way. In particular, we study a uniformly accelerated source of a quantum field state-a single-mode squeezer. Even though the initial state of the field is vacuum (a pure state) and the interaction with the quantum source in the accelerated frame is unitary, we find that the final state detected by inertial observers appears to be decohered, i.e., in a mixed state. This unexpected result may indicate new directions in resolving inconsistencies between relativity and quantum theory. We extend this result to a two-mode state and find that entanglement is also decohered.
\end{abstract}

DOI: 10.1103/PhysRevX.9.011007

Subject Areas: Quantum Physics

\section{INTRODUCTION}

Unitary evolution is one of the fundamental assumptions of quantum mechanics. An initial pure state of an isolated quantum system always evolves into another pure state. The situation is not as simple when we consider noninertial, relativistic frames of reference. For example, the transformation between the description of the quantum vacuum state as seen by inertial observers and the description of the same state by uniformly accelerated observers is not strictly unitary. Nevertheless, it is still assumed that in transforming between reference frames, pure states will always evolve to pure states, provided that the entire space-time is included.

Consider inertial observers who constantly observe a massless field prepared in the Minkowski vacuum state. By definition, they will observe no particles. However, according to the Unruh-Davies effect [1,2], a uniformly accelerating observer who constantly observes the same field will see thermal radiation (Unruh radiation) and hence will count particles. The vacuum state is pure, while a thermal state is mixed, seemingly implying a nonunitary evolution. The resolution is that a single accelerating observer is restricted to a section of space-time called a Rindler wedge.

\footnotetext{
"sudaiqin@gmail.com

†ralph@physics.uq.edu.au
}

Published by the American Physical Society under the terms of the Creative Commons Attribution 4.0 International license. Further distribution of this work must maintain attribution to the author(s) and the published article's title, journal citation, and DOI.
By introducing a second, mirror-image, accelerated observer, we find that the thermal state can be purified into a two-mode squeezed state [3-5], and unitarity is restored.

Because of the equivalence principle, there is a strong relationship between gravity and acceleration [6]. The analogous situation to Unruh radiation in curved spacetime is that of thermal radiation from black holes (Hawking radiation) [7]. In this case, regaining unitarity is not straightforward because the analogue of the mirror image Rindler wedge lies behind the black hole event horizon and so is inaccessible. Given that in the far future the black hole is expected to completely evaporate, this leads to the black hole information paradox [8]. In spite of many attempts [9-13], a completely satisfactory resolution of this problem has not been found $[14,15]$.

In this paper, we consider quantum systems in flat space; however, we set up the problem differently such that we explicitly start and end with global, inertial observers. In the intermediate region, we allow single-mode interactions with an accelerated system. Our inertial observers use "bucket" detectors to observe all frequencies. In contrast to previous treatments, this model leads to exact, semianalytical solutions. Unexpectedly, we find a decoherence effect that only affects nonclassical quantum states and appears even though the observer's detection technique has access to the entire space-time.

Consider the following situation. Alice produces coherent states and sends them to Bob. Bob directly detects them with a coarse-grained intensity detector which is sensitive to a range of frequencies much larger than those contained in the pulses. In principle, Bob can detect the intensity of 
the sent pulses with a precision given by the shot-noise (or quantum-noise) variance. This idea is the basis of incoherent optical communication. It is well known that if Alice squeezes her coherent pulses, then Bob can detect the intensity of the pulses with increased precision, which is subshot noise. Here, we show that if Alice is accelerating at a sufficient rate, it becomes impossible for her to send pulses with non-negligible squeezing to Bob in this scenario.

The specific problem we analyze is summarized by the Penrose diagram [6] in Fig. 1. An object uniformly accelerates in the right Rindler wedge (black curve). Interactions with a massless scalar field are unitarily turned on and off during its lifetime (shaded region) such that it interacts with a single spatiotemporal mode in the accelerated (Rindler) coordinates. In the past null infinity $\mathcal{I}^{-}$, the initial state of the field is set as the Minkowski vacuum. For simplicity, we consider a $1+1$ theory in which the rightand left-moving fields are decoupled [16]. We assume the right-moving field modes are unaffected by the accelerating object. The output state of the left-moving field modes in the future null infinity $\mathcal{I}^{+}$is detected by inertial, Minkowski detectors. We ask whether the detected field statistics are those of a pure state.

In the following section, we introduce our detection model. In Sec. III, we introduce our source model and solve for the detection statistics of a single-mode coherent state (Sec. III A) and a squeezed state (Sec. III B). In Sec. III C, we extend this picture to two-mode sources that entangle

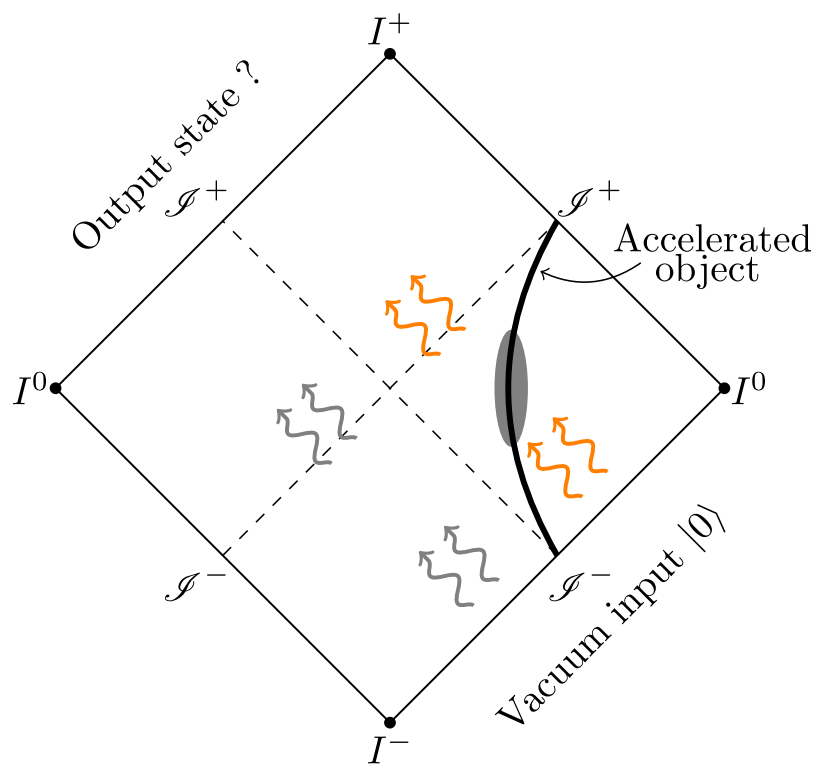

FIG. 1. Penrose diagram of Minkowski spacetime. Here, $I^{0}$ is the spatial infinity, $I^{-}$and $I^{+}$are the past and future infinities, and $\mathcal{I}^{-}$and $\mathcal{I}^{+}$are the past and future null infinities. A uniformly accelerated object follows the black worldline. Interactions between the accelerated object and the field are localized in Rindler time, represented by the shaded region. right and left movers. In Sec. IV, we discuss the origin of the decoherence effect, resolve the apparent paradox between the unitary source and the mixed statistics, and draw possible connections with black-hole physics.

\section{DETECTION OF THE STATE}

The Minkowski detectors are modeled by the Hermitian number operators, $\hat{N}_{k}=\hat{a}_{k}^{\dagger} \hat{a}_{k}$, where $\hat{a}_{k}\left(\hat{a}_{k}^{\dagger}\right)$ are the Minkowski field annihilation (creation) operators for wave number $k$. The frequencies $\Omega=|k|$ are with respect to the proper time of the inertial reference frame under consideration (note we are using units for which $c=1$ ). The excitation probability of an ideal, inertial, two-level system of resonant frequency $\Omega$, coupled weakly to the field, is proportional to $\left\langle\hat{N}_{k}\right\rangle$ [17]. We can model a finite-bandwidth detector via the operator $\hat{N}_{\Delta k}=\int_{k_{o}-\Delta k}^{k_{o}+\Delta k} \mathrm{~d} k \hat{a}_{k}^{\dagger} \hat{a}_{k}$. If the bandwidth of the detector is much larger than that of the mode under consideration, then we can extend the limits of integration to $\pm \infty$ and thus define $\hat{N}=\int \mathrm{d} k \hat{a}_{k}^{\dagger} \hat{a}_{k}$. Note that, by definition, $\langle 0|\hat{N}| 0\rangle=0$ for the Minkowski vacuum state $|0\rangle$.

In order to characterize the state of a particular field mode, we use homodyne tomography [18]. In homodyne tomography, the Wigner function [17] of the state is reconstructed from measurements of the moments of quadrature amplitudes via homodyne detection. For Gaussian states, it is sufficient to measure and analyze only the first- and second-order moments [19]. In homodyne detection [20], a weak signal field and a strong local oscillator are coherently combined and measured with broadband detection as discussed above. For simplicity and to stay within the $1+1$ scalar field paradigm, we specifically use self-homodyne detection here. In selfhomodyne detection, the signal field is displaced by a strong local oscillator directly, and the output field is detected. Assume that the signal field mode operator is $\hat{a}=\int \mathrm{d} k f(k) \hat{a}_{k}$ and the local oscillator is a strong coherent state $|\alpha\rangle$, prepared in the same field mode [characterized by $f(k)]$ with $\alpha$ a complex number, $\alpha=|\alpha| e^{i \phi}$, and $|\alpha| \gg 1$. The photon number operator can be shown to be

$$
\hat{N}(\phi) \approx|\alpha|^{2}+|\alpha| \hat{X}(\phi),
$$

where $\hat{X}(\phi)=\hat{a} e^{-i \phi}+\hat{a}^{\dagger} e^{i \phi}$ is the quadrature amplitude of the signal field and a term not multiplied by $|\alpha|$ has been neglected as it is much smaller than other terms. As a reference, we can also consider the operator

$$
\hat{N}_{0} \approx|\alpha|^{2}+|\alpha| \hat{X}_{v}
$$

representing the situation where the signal is not imposed, so $\hat{v}$ represents the mode when it is prepared in the vacuum state. Hence, the average quadrature amplitude of the field is given by 


$$
\langle\hat{X}(\phi)\rangle=\frac{\langle\hat{N}(\phi)\rangle-\left\langle\hat{N}_{0}\right\rangle}{\sqrt{\left\langle\hat{N}_{0}\right\rangle}},
$$

where we have used $\left\langle\hat{X}_{v}\right\rangle=0$. Its variance is given by

$$
(\Delta X(\phi))^{2}=\frac{(\Delta N(\phi))^{2}}{\left\langle\hat{N}_{0}\right\rangle} .
$$

For the Gaussian states considered here, this will be sufficient to completely characterize them. We wish to apply this technique to the output state from the interactions between a uniformly accelerated object and the scalar field. In order to match the mode shape of the local oscillator to that of the output signal field, we assume that the local oscillator is also imposed in the accelerated frame in a matching mode to the signal.

\section{INTERACTION WITH THE ACCELERATED SOURCE}

Interactions between uniformly accelerated objects (Unruh-DeWitt detectors, mirrors, etc.) and quantum fields have been studied for many years [21-24]. Recently, a nonperturbative quantum circuit model was proposed to investigate these interactions and calculate radiation from a uniformly accelerated object [25]. Here, we generalize the circuit model to include time-dependent interactions. The relevant circuit is shown in Fig. 2. The circuit models the interaction as a Heisenberg evolution of Unruh mode operators [1] $\hat{c}_{\omega}, \hat{d}_{\omega}$ to Rindler operators $\hat{b}_{\omega}^{L}, \hat{b}_{\omega}^{R}$, then back to Unruh operators. The Rindler operators represent the natural modes that uniformly accelerated systems interact

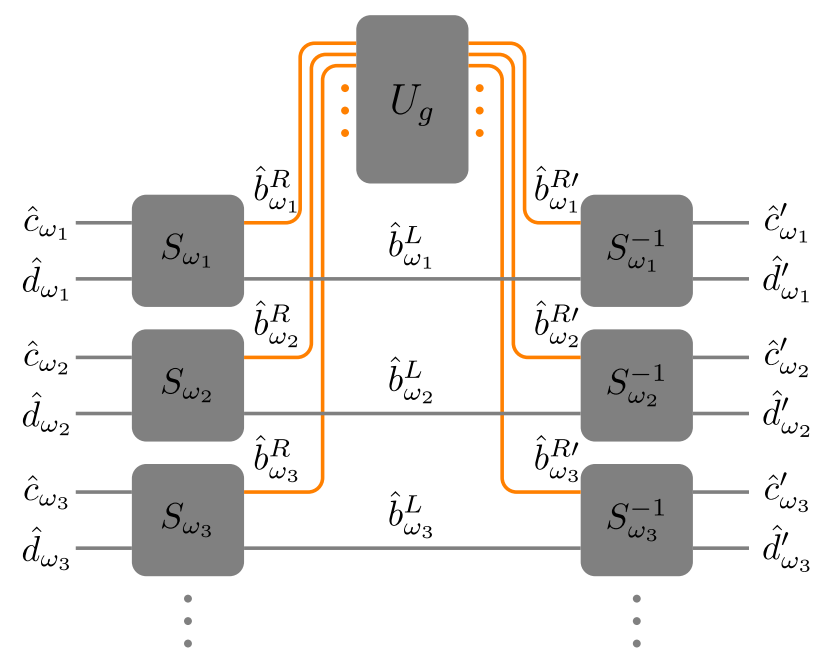

FIG. 2. Circuit for a uniformly accelerated object. Rindler modes in the right Rindler wedge interact with the object, which is represented by the unitary operator $\hat{U}_{g}$, while Rindler modes in the left Rindler wedge remain unaffected. The time-dependent interactions mix different frequency Rindler modes. with. The frequency $\omega$ is with respect to the proper time of the accelerated observer. The Unruh operators are a useful mathematical stepping stone between the accelerated and inertial reference frames. The Minkowski modes $\hat{a}_{k}$ that represent our inertial detection scheme are then constructed from the output Unruh modes-this final step is not represented by a circuit.

The unitary operator $\hat{U}_{g}$ acts only on the right Rindler wedge operators $\hat{b}_{\omega}^{R}$ and represents localized interactions between the accelerated object and the scalar field. The localization is characterized by the normalized wave packet $g(\omega)$. In contrast to the time-independent case, the timedependent unitary $\hat{U}_{g}$ mixes different Rindler frequency modes. The relation between the Rindler modes $\hat{b}_{\omega}^{R \prime}$ and $\hat{b}_{\omega}^{R}$ is [26]

$$
\hat{b}_{\omega}^{R \prime}=\hat{b}_{\omega}^{R}+g^{*}(\omega)\left(\hat{U}_{g}^{\dagger} \hat{b}_{g}^{R} \hat{U}_{g}-\hat{b}_{g}^{R}\right),
$$

where $\hat{b}_{g}^{R} \equiv \int \mathrm{d} \omega g(\omega) \hat{b}_{\omega}^{R}$ is the localized mode operator satisfying commutation relation $\left[\hat{b}_{g}^{R}, \hat{b}_{g}^{R^{\dagger}}\right]=1$. We note that $\left[\hat{b}_{\omega}^{R}, \hat{b}_{\omega^{\prime}}^{R \dagger}\right]=\left[\hat{b}_{\omega}^{R \prime}, \hat{b}_{\omega^{\prime}}^{R \prime \dagger}\right]=\delta\left(\omega-\omega^{\prime}\right)$, indicating that Eq. (5) is a unitary interaction. Taking into account the relation between Unruh modes and Rindler modes [25], which is basically a two-mode squeezing, we obtain the input-output relations for Unruh modes,

$$
\begin{aligned}
& \hat{c}_{\omega}^{\prime}=\hat{c}_{\omega}+g^{*}(\omega) \cosh r_{\omega}\left(\hat{U}_{g}^{\dagger} \hat{b}_{g}^{R} \hat{U}_{g}-\hat{b}_{g}^{R}\right), \\
& \hat{d}_{\omega}^{\prime}=\hat{d}_{\omega}-g(\omega) \sinh r_{\omega}\left(\hat{U}_{g}^{\dagger} \hat{b}_{g}^{R^{\dagger}} \hat{U}_{g}-\hat{b}_{g}^{R^{\dagger}}\right),
\end{aligned}
$$

where the two-mode squeezing factor $r_{\omega}$ is defined as $\tanh r_{\omega}=e^{-\pi \omega / a}$. In Eq. (6), the operator $\hat{b}_{g}^{R}$ can be explicitly expressed in terms of the input Unruh modes $\hat{c}_{\omega}$ and $\hat{d}_{\omega}$. In the following, we use $\hat{U}_{g}=\hat{D}_{g}(\alpha) \hat{S}_{g}$, where $\hat{S}_{g}$ creates the quantum signal we wish to analyze, while $\hat{D}_{g}(\alpha)=\exp \left(\alpha \hat{b}_{g}^{R \dagger}-\alpha^{*} \hat{b}_{g}^{R}\right)$ produces the local oscillator needed for the self-homodyne detection (Fig. 3). It is easy to show that $\hat{D}_{g}^{\dagger} \hat{b}_{g}^{R} \hat{D}_{g}=\hat{b}_{g}^{R}+\alpha$ [20].

Finally, we require the input-output relations for Minkowski modes. The transformation from Unruh modes to Minkowski modes is [25]

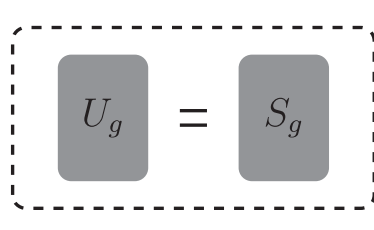

(a)

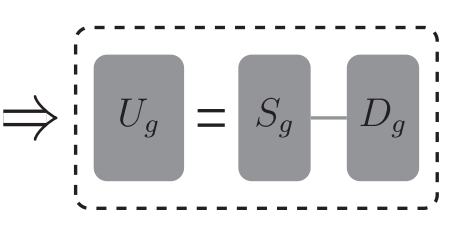

(b)
FIG. 3. Self-homodyne detection. (a) A signal unitary $\hat{S}_{g}$ generates the quantum signals that we are going to analyze. (b) A displacement is added after the signal unitary $\hat{S}_{g}$ to realize homodyne detection. The mode shape of the displacement is perfectly matched to that of the signal unitary. 


$$
\hat{a}_{k}^{\prime}=\int \mathrm{d} \omega\left(A_{k \omega} \hat{c}_{\omega}^{\prime}+B_{k \omega} \hat{d}_{\omega}^{\prime}\right)
$$

where the Bogoliubov transformation coefficients are [16]

$$
A_{k \omega}=B_{k \omega}^{*}=\frac{i \sqrt{2 \sinh (\pi \omega / a)}}{2 \pi \sqrt{\omega k}} \Gamma(1-i \omega / a)\left(\frac{k}{a}\right)^{i \omega / a} .
$$

The total Minkowski particle number operator is obtained by using Eq. (7),

$$
\begin{aligned}
\hat{N}= & \int \mathrm{d} k \int \mathrm{d} \omega_{1} \int \mathrm{d} \omega_{2}\left(A_{k \omega_{1}}^{*} \hat{c}_{\omega_{1}}^{\prime \dagger}+B_{k \omega_{1}}^{*} \hat{d}_{\omega_{1}}^{\prime \dagger}\right) \\
& \times\left(A_{k \omega_{2}} \hat{c}_{\omega_{2}}^{\prime}+B_{k \omega_{2}} \hat{d}_{\omega_{2}}^{\prime}\right) \\
= & \int \mathrm{d} \omega\left(\hat{c}_{\omega}^{\prime \dagger} \hat{c}_{\omega}^{\prime}+\hat{d}_{\omega}^{\prime \dagger} \hat{d}_{\omega}^{\prime}\right),
\end{aligned}
$$

where we have used $\int \mathrm{d} k A_{k \omega} A_{k \omega^{\prime}}^{*}=\delta\left(\omega-\omega^{\prime}\right)$ and $\int \mathrm{d} k A_{k \omega} A_{k \omega^{\prime}}=0$. The square of the total particle number operator is

$$
\begin{aligned}
\hat{N}^{2}= & \int \mathrm{d} \omega_{1} \int \mathrm{d} \omega_{2}\left(\hat{c}_{\omega_{1}}^{\prime \dagger} \hat{c}_{\omega_{1}}^{\prime} \hat{c}_{\omega_{2}}^{\prime \dagger} \hat{c}_{\omega_{2}}^{\prime}+\hat{d}_{\omega_{1}}^{\prime \dagger} \hat{d}_{\omega_{1}}^{\prime} \hat{d}_{\omega_{2}}^{\prime \dagger} \hat{d}_{\omega_{2}}^{\prime}\right. \\
& \left.+\hat{c}_{\omega_{1}}^{\prime \dagger} \hat{c}_{\omega_{1}}^{\prime} \hat{d}_{\omega_{2}}^{\prime \dagger} \hat{d}_{\omega_{2}}^{\prime}+\hat{d}_{\omega_{1}}^{\prime \dagger} \hat{d}_{\omega_{1}}^{\prime} \hat{c}_{\omega_{2}}^{\prime \dagger} \hat{c}_{\omega_{2}}^{\prime}\right) .
\end{aligned}
$$

A full computation of the vacuum expectation value of $\hat{N}^{2}$ is straightforward but usually tedious. However, when the amplitude of displacement is large $(|\alpha| \gg 1)$, it is adequate to only keep terms of order $|\alpha|^{4}$ and $|\alpha|^{2}$ as per the approximation leading to Eqs. (3) and (4).

\section{A. Classical signals}

We first consider preparing a classical signal on the accelerated mode. In particular, we generate a classical signal by displacing the Rindler mode $\hat{b}_{g}^{R}$ with an amplitude $\beta$. This produces a coherent state, the "most classical" quantum state. The operator that creates this signal is $\hat{S}_{g}=\hat{D}_{g}(\beta)$, with $|\beta| \ll|\alpha|$. The expectation value and variance of the quadrature amplitudes as observed by the inertial detectors are

$$
\begin{aligned}
& X_{\beta}(\phi)=\sqrt{\mathcal{I}_{c}+\mathcal{I}_{s}}\left(\beta e^{-i \phi}+\beta^{*} e^{i \phi}\right), \\
& V_{\beta}(\phi)=1,
\end{aligned}
$$

where $\mathcal{I}_{c}=\int \mathrm{d} \omega|g(\omega)|^{2} \cosh ^{2} r_{\omega}$ and $\mathcal{I}_{s}=\int \mathrm{d} \omega|g(\omega)|^{2}$ $\sinh ^{2} r_{\omega}$. Equation (11) characterizes a pure coherent state. Therefore, displacing a Rindler mode generates a coherent state with amplitude $\left(\sqrt{\mathcal{I}_{c}+\mathcal{I}_{s}}\right) \beta$ as viewed by an inertial observer. As expected, the overall evolution is from a pure state to a pure state.

\section{B. Quantum signals}

A more interesting scenario is that a uniformly accelerated single-mode squeezer squeezes the thermal state in the right Rindler wedge. The single-mode squeezing operator $\hat{S}_{1}(r)$ is defined as [20]

$$
\hat{S}_{1}(r)=\exp \left\{\frac{r}{2}\left(\hat{b}_{g}^{R^{\dagger}}\right)^{2}-\frac{r}{2}\left(\hat{b}_{g}^{R}\right)^{2}\right\},
$$

where $r$ is the squeezing factor and is assumed to be real. The operator that creates quantum signals is $\hat{S}_{g}=\hat{S}_{1}(r)$ so that the unitary $\hat{U}_{g}=\hat{D}_{g}(\alpha) \hat{S}_{1}(r)$. By substituting this unitary into Eq. (6), one can derive the input-output relations for Unruh modes, which are then substituted into Eqs. (9) and (10) to calculate the vacuum expectation value of the Minkowski particle number and the square of the particle number (see Appendix for details). We find that the expectation value of the quadrature amplitude is zero, and the variance is

$$
\begin{aligned}
V(\phi)= & \cosh (2 r)+4 \mathcal{I}_{c}\left(\mathcal{I}_{c}-1\right)(\cosh 2 r-2 \cosh r+1) \\
& +2 \sinh r\left[\left(2 \mathcal{I}_{c}-1\right)^{2} \cosh r-4 \mathcal{I}_{c}\left(\mathcal{I}_{c}-1\right)\right] \cos (2 \phi) .
\end{aligned}
$$

The maximum and minimum variances are obtained when $\phi=0$ and $\phi=\pi / 2$, respectively:

$$
\begin{aligned}
& V_{\text {max }}=e^{2 r}+4 \mathcal{I}_{c}\left(\mathcal{I}_{c}-1\right)\left(e^{r}-1\right)^{2}, \\
& V_{\text {min }}=e^{-2 r}+4 \mathcal{I}_{c}\left(\mathcal{I}_{c}-1\right)\left(e^{-r}-1\right)^{2} .
\end{aligned}
$$

It is evident from Eqs. (13) and (14) that noises are added onto the variance of the original single-mode squeezed state. The amount of additional noises depends on the squeezing factor $r$ and $\mathcal{I}_{c}$. A question of particular interest is whether the final state is a pure state. For Gaussian states, the criterion for purity is that the product of maximum and minimum variances is unity [20]. From Eq. (14), we find the product of the maximum and minimum variances is

$$
\begin{aligned}
V_{\max } V_{\min }= & 1+16 \mathcal{I}_{c}\left(\mathcal{I}_{c}-1\right)(\cosh r-1) \cosh r \\
& +64 \mathcal{I}_{c}^{2}\left(\mathcal{I}_{c}-1\right)^{2}(\cosh r-1)^{2} .
\end{aligned}
$$

We can see that the product is always greater than 1 unless $r=0$ or $\mathcal{I}_{c}=1$. This is our main result. Unexpectedly, the inertial observer sees a decoherence effect that, in general, causes the initial pure state to display mixed state statistics.

The case of $r=0$ means that the accelerated object does nothing, so the output state is the Minkowski vacuum. Note that $\mathcal{I}_{c}$ can be approximated as $\mathcal{I}_{c} \approx e^{2 \pi \omega_{0} / a} /\left(e^{2 \pi \omega_{0} / a}-1\right)$ when $g(\omega)$ is a very-narrow-bandwidth wave packet with central frequency $\omega_{0}$. When $2 \pi \omega_{0} / a \rightarrow \infty, \mathcal{I}_{c} \rightarrow 1$, so $V_{\min } \rightarrow e^{-2 r}$ and $V_{\max } \rightarrow e^{2 r}$. This corresponds to a 
single-mode squeezed vacuum state, which is pure. The above limit could happen in two cases. The first is that the central frequency $\omega_{0}$ is fixed while $a \rightarrow 0$. This means the single-mode squeezer tends to be static in an inertial frame. It thus produces the standard single-mode squeezed vacuum state. The second case is that $a$ is fixed and finite, while $\omega_{0} \rightarrow \infty$. It is well known that a uniformly accelerated observer experiences a thermal radiation with temperature $T_{U}=[a /(2 \pi)]$ in the Minkowski vacuum [1]. The spectral distribution of the thermal radiation follows Plank's law, which exponentially decays in the high-frequency limit. Or equivalently, the high-frequency tail of a thermal state looks almost like a vacuum. Therefore, the single-mode squeezer that squeezes the high-frequency tail of the Unruh radiation produces a squeezed vacuum state. Overall, when the Unruh effect is not significant, a uniformly accelerated single-mode squeezer produces the standard single-mode squeezed vacuum state. Otherwise, the product of the maximum and minimum variances is greater than 1 , indicating that the output state is mixed.

As the Unruh effect in the Rindler frame becomes more pronounced, the decoherence in the Minkowski frame becomes stronger. Eventually, squeezing disappears and the final state becomes classical in the sense that coherent
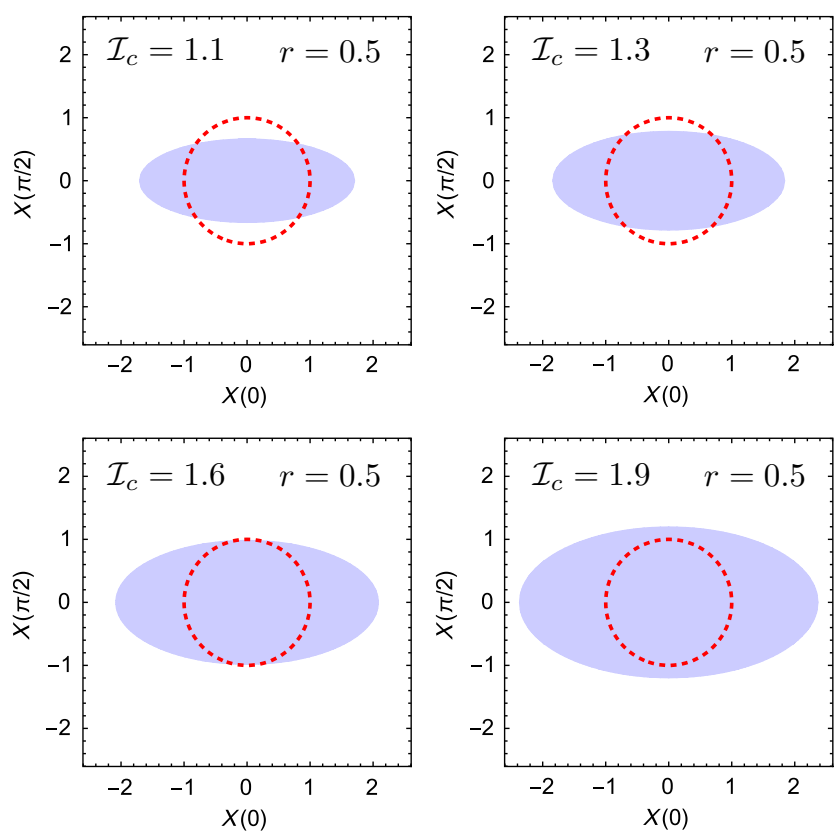

FIG. 4. Phase-space representation of quadrature in the final state. The red dashed circle represents the vacuum shot noise, and the blue shaded ellipse represents the quadrature variance of the output state. For a fixed single-mode squeezing factor $(r=0.5)$, the minimum quadrature variance is below the vacuum shot noise for small $\mathcal{I}_{c}$, indicating that the output state is a squeezed state. While for large enough $\mathcal{I}_{c}$, the minimum quadrature variance surpasses the vacuum shot noise, showing that squeezing is destroyed.

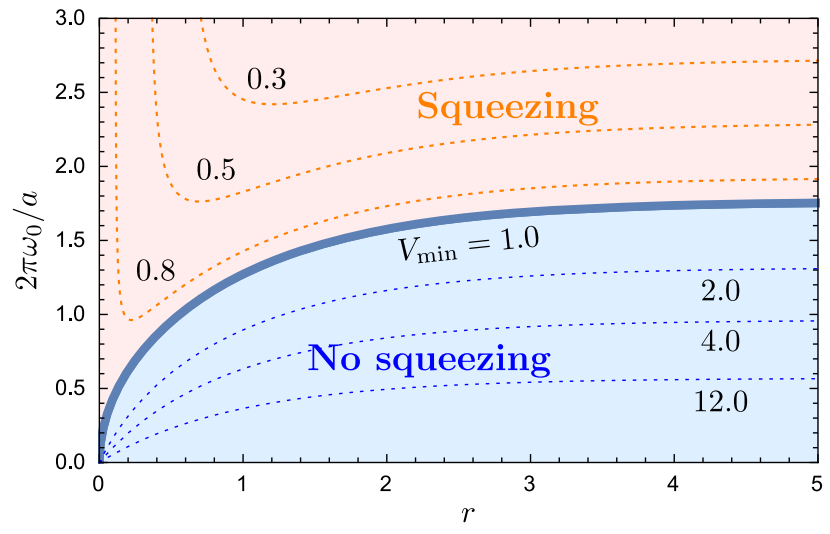

FIG. 5. Distribution of minimum quadrature variance of the output state as a function of single-mode squeezing factor $r$ and the central frequency $\omega_{0}$ in the narrow-bandwidth limit. A critical curve along which $V_{\min }=1.0$ separates the squeezing region and no-squeezing region. In the squeezing region, $V_{\min }<1.0$, while in the no-squeezing region, $V_{\min }>1.0$.

state superpositions are removed, and the state becomes decomposable into a mixture of coherent states. Figure 4 shows an example of the phase-space representation of the quadrature amplitude. In the narrow-bandwidth limit, we use the approximate relation between $\mathcal{I}_{c}$ and $\omega_{0}$ to find the distribution of minimum quadrature variance in terms of $r$ and $\omega_{0}$, as shown in Fig. 5. A critical curve, which is determined by

$$
\frac{2 \pi \omega_{0}}{a}=\ln \left(\frac{\sqrt{1+\operatorname{coth}(r / 2)}+1}{\sqrt{1+\operatorname{coth}(r / 2)}-1}\right)
$$

separates the squeezing region and no-squeezing region. When $r \rightarrow \infty, 2 \pi \omega_{0} / a \rightarrow 2 \ln (\sqrt{2}+1) \approx 1.763$. Below this value, one can always make the output state classical by increasing the single-mode squeezing factor $r$. Put another way, as claimed in the Introduction, for a sufficiently small value of $\left[\left(2 \pi \omega_{0}\right) / a\right]$, it becomes impossible to produce any significant squeezing of the intensity fluctuations detected by the inertial observer.

\section{Entanglement results}

We generalize the above calculation to a uniformly accelerated two-mode squeezer in the right Rindler wedge that couples the left-moving and right-moving Rindler modes. The two-mode squeezing operator is defined as [17]

$$
\hat{S}_{2}(r)=\exp \left\{r\left(\hat{b}_{1 g}^{R^{\dagger}} \hat{b}_{2 g}^{R^{\dagger}}-\hat{b}_{1 g}^{R} \hat{b}_{2 g}^{R}\right)\right\}
$$

where the subscripts "1" and "2" represent the left-moving and right-moving modes, respectively. Here, $r$ is the squeezing factor and is assumed to be real. The output field includes the left-moving and right-moving parts. To have full information about the output state, one needs to 
measure the states of the left-moving and right-moving modes, as well as the correlations between them.

We add two displacements, with amplitudes $\alpha_{1}=$ $\left|\alpha_{1}\right| e^{i \phi_{1}}$ and $\alpha_{2}=\left|\alpha_{2}\right| e^{i \phi_{2}}$, after the two-mode squeezer in order to perform homodyne detection, the former for the left-moving mode and the latter for the right-moving mode. We find that the expectation values of the quadrature amplitudes $\hat{X}_{1}\left(\phi_{1}\right)$ and $\hat{X}_{2}\left(\phi_{2}\right)$ are zero. The covariance matrix [19] of the output state is

$$
\mathbf{V}=\left(\begin{array}{cccc}
\mathcal{A} & 0 & \mathcal{B} & 0 \\
0 & \mathcal{A} & 0 & -\mathcal{B} \\
\mathcal{B} & 0 & \mathcal{A} & 0 \\
0 & -\mathcal{B} & 0 & \mathcal{A}
\end{array}\right)
$$

where

$$
\begin{aligned}
& \mathcal{A}=\left(2 \mathcal{I}_{c}-1\right)^{2} \cosh (2 r)-4 \mathcal{I}_{c}\left(\mathcal{I}_{c}-1\right)(2 \cosh r-1), \\
& \mathcal{B}=2 \sinh r\left[\left(2 \mathcal{I}_{c}-1\right)^{2} \cosh r-4 \mathcal{I}_{c}\left(\mathcal{I}_{c}-1\right)\right] .
\end{aligned}
$$

From the covariance matrix (18), one can derive the logarithmic negativity as [19]

$$
E_{\mathcal{N}}=\max \left[0,-\log _{2}\left(\tilde{\nu}_{-}\right)\right]
$$

where $\tilde{\nu}_{-}$is the smallest symplectic eigenvalue of the partially transposed state,

$$
\tilde{\nu}_{-}=e^{-2 r}+4 \mathcal{I}_{c}\left(\mathcal{I}_{c}-1\right)\left(e^{-r}-1\right)^{2} .
$$

When $\tilde{\nu}_{-}<1\left(E_{\mathcal{N}}>0\right)$, there exists entanglement between the left-moving and right-moving modes; when $\tilde{\nu}_{-} \geq 1$ $\left(E_{\mathcal{N}}=0\right)$, the left-moving and right-moving modes are not entangled. When $\mathcal{I}_{c}=1$, the covariance matrix [Eq. (18)]

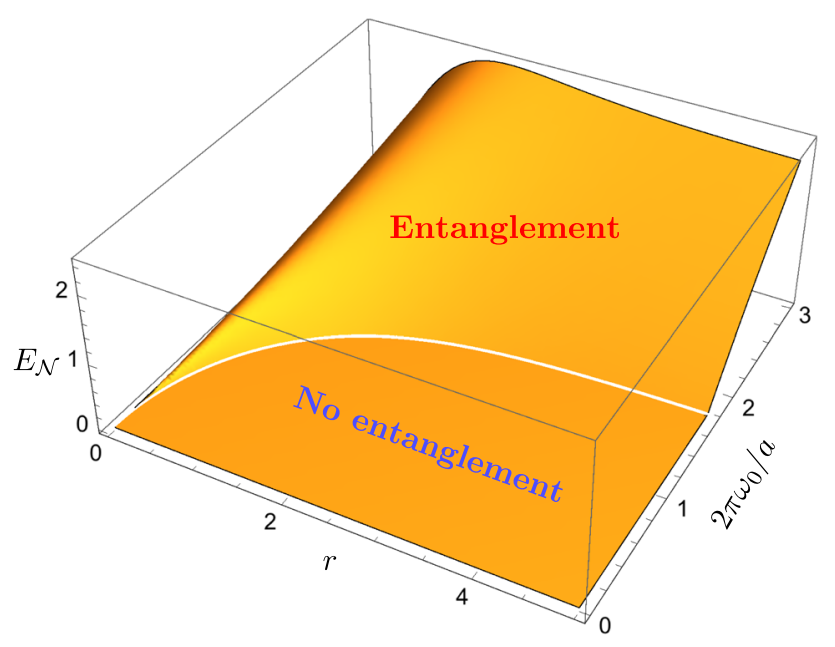

FIG. 6. Logarithmic negativity of the output state as a function of the squeezing factor $r$ and the central frequency $\omega_{0}$ in the narrow-bandwidth limit. is that of a pure two-mode squeezed state and the entanglement [Eq. (21)] is maximized. However, when $\mathcal{I}_{c}>1$, the covariance matrix becomes decohered (mixed) and the entanglement drops, eventually disappearing. Figure 6 shows the logarithmic negativity as a function of the squeezing factor $r$ and the central frequency $\omega_{0}$ in the narrow-bandwidth limit. The critical curve $\tilde{\nu}_{-}=1$, dividing the entanglement and no-entanglement regions, is determined by Eq. (16).

\section{CONCLUSION}

The decoherence effect we describe here is a previously unnoticed consequence of the transformation from the bipartite Hilbert space of the Rindler and Unruh modes to the single Hilbert space of the Minkowski modes. Notice that in Eq. (9), any direct phase relationship between the left and right Unruh modes is lost in the construction of the Minkowski number operator. This means that interactions that lead to entanglement between the left and right Unruh modes, as occurs with the accelerated squeezer and the entangler, will, in general, appear as decoherence with respect to measurements by inertial observers. In contrast, coherent state signals do not produce Unruh mode entanglement, so no decoherence is observed for such signals.

We note that it is quite standard for the LO to be sent from the source as a phase or mode reference along with the signal. In direct communication scenarios, the local oscillator (i.e., the coherent amplitude) is mixed with the signal from the start (self-homodyne). In principle, the LO could be sent separately from the signal, and the inertial observer could carry out its own homodyne by mixing the LO with the signal locally. However, in the accelerated case, this only works if the phase relationship between the LO and the signal (established by Alice) is maintained by Bob. If Bob introduces his own Minkowski phase shift (by producing a delay between the LO and signal), then the measurement result will be scrambled, and even coherent states will look mixed. Thus, in order to see pure coherent state signals in a practical scenario, Alice and Bob should employ the self-homodyne approach for their communication. Surprisingly, this technique then fails to see pure state statistics when nonclassical (squeezed) states are used.

We have shown that single- and two-mode unitary squeezing operations in an accelerated frame are, in general, detected as decohered states by inertial observers. As we noted in the Introduction, the standard Unruh effect can be purified if a mirror-image accelerated observer is introduced. Here, we find that a mirror-image accelerated source is required to purify the state detected by the inertial observer. In particular, for the narrow-band case, if the mirror-image source displaces the state by $\gamma=$ $2\left\{\left[\sqrt{\mathcal{I}_{c}\left(\mathcal{I}_{c}-1\right)}\right] /\left(2 \mathcal{I}_{c}-1\right)\right\} \alpha^{*}$, in phase with the original accelerated source, then the inertial detectors will see pure states in both the squeezer and entangler cases. Details of this calculation are given in the Appendix. 
We believe the decoherence effect has significance for understanding quantum effects in gravitational systems. For example, our system can be viewed as a toy model for the creation and eventual evaporation of a black hole. We begin in the far past in a pure Minkowski vacuum state before the formation of the black hole. In the intermediate epoch, accelerated observers, representing observers close to the black hole, interact with the field modes. Finally, in the far future, the black hole has evaporated, leaving flat space; however, the field remains in a mixed state with respect to inertial observers. This may indicate a new direction for understanding the black-hole information paradox.

The discovered decoherence effect could, in principle, be used to detect and measure the acceleration between inertial and accelerated observers. However, the accelerations required to generate this decoherence effect are well beyond those that can be physically produced in the lab. On the other hand, such accelerations occur naturally in many regions of the Universe. In addition, the equivalence between acceleration and time-dependent effects [27] may enable laboratory tests, especially at microwave frequencies [28]. We also note that simulation of these effects using optical squeezing is possible with current technology and would allow an experimental investigation of analogues to the decoherence effect described here that may be of interest in their own right from a quantum information point of view.

\section{ACKNOWLEDGMENTS}

We thank Robert Mann for useful comments. This research was supported by Australian Research Council Centre of Excellence of Quantum Computation and Communication Technology (Project No. CE170100012).

\section{APPENDIX A: DERIVATION OF THE VARIANCE IN SINGLE-MODE SQUEEZING CASE}

In the single-mode squeezing case, the unitary is taken to be $\hat{U}_{g}=\hat{D}_{g}(\alpha) \hat{S}_{1}(r)$. By substituting this unitary into Eq. (6), one can derive the input-output relations for Unruh modes,

$\hat{c}_{\omega}^{\prime}=\hat{c}_{\omega}+g^{*}(\omega) \cosh r_{\omega}\left[\hat{b}_{g}^{R}(\cosh r-1)+\hat{b}_{g}^{R^{\dagger}} \sinh r+\alpha\right]$, $\hat{d}_{\omega}^{\prime}=\hat{d}_{\omega}-g(\omega) \sinh r_{\omega}\left[\hat{b}_{g}^{R^{\dagger}}(\cosh r-1)+\hat{b}_{g}^{R} \sinh r+\alpha^{*}\right]$.

The localized Rindler operator $\hat{b}_{g}^{R}$ can be expressed in terms of the input Unruh operators by using the transformations between the Rindler and Unruh modes. Equation (A1) becomes

$$
\begin{aligned}
\hat{c}_{\omega}^{\prime}= & \hat{c}_{\omega}+g^{*}(\omega) \cosh r_{\omega}\left[(\cosh r-1) \int \mathrm{d} \omega^{\prime} g\left(\omega^{\prime}\right)\left(\hat{c}_{\omega^{\prime}} \cosh r_{\omega^{\prime}}+\hat{d}_{\omega^{\prime}}^{\dagger} \sinh r_{\omega^{\prime}}\right)\right. \\
& \left.+\sinh r \int \mathrm{d} \omega^{\prime} g^{*}\left(\omega^{\prime}\right)\left(\hat{c}_{\omega^{\prime}}^{\dagger} \cosh r_{\omega^{\prime}}+\hat{d}_{\omega^{\prime}} \sinh r_{\omega^{\prime}}\right)+\alpha\right], \\
\hat{d}_{\omega}^{\prime}= & \hat{d}_{\omega}-g(\omega) \sinh r_{\omega}\left[(\cosh r-1) \int \mathrm{d} \omega^{\prime} g^{*}\left(\omega^{\prime}\right)\left(\hat{c}_{\omega^{\prime}}^{\dagger} \cosh r_{\omega^{\prime}}+\hat{d}_{\omega^{\prime}} \sinh r_{\omega^{\prime}}\right)\right. \\
& \left.+\sinh r \int \mathrm{d} \omega^{\prime} g\left(\omega^{\prime}\right)\left(\hat{c}_{\omega^{\prime}} \cosh r_{\omega^{\prime}}+\hat{d}_{\omega^{\prime}}^{\dagger} \sinh r_{\omega^{\prime}}\right)+\alpha^{*}\right] .
\end{aligned}
$$

It is now straightforward to calculate the vacuum expectation values of the product of two output Unruh operators:

$$
\begin{aligned}
& \left\langle 0\left|\hat{c}_{\omega}^{\dagger} \hat{c}_{\omega^{\prime}}^{\prime}\right| 0\right\rangle=g(\omega) g^{*}\left(\omega^{\prime}\right) \cosh r_{\omega} \cosh r_{\omega^{\prime}}\left(E_{c}+|\alpha|^{2}\right), \\
& \left\langle 0\left|\hat{d}_{\omega}^{\prime \dagger} \hat{d}_{\omega^{\prime}}^{\prime}\right| 0\right\rangle=g^{*}(\omega) g\left(\omega^{\prime}\right) \sinh r_{\omega} \sinh r_{\omega^{\prime}}\left(E_{d}+|\alpha|^{2}\right) \text {, } \\
& \left\langle 0\left|\hat{c}_{\omega}^{\prime} \hat{c}_{\omega^{\prime}}^{\prime}\right| 0\right\rangle=g^{*}(\omega) g^{*}\left(\omega^{\prime}\right) \cosh r_{\omega} \cosh r_{\omega^{\prime}}\left(E_{c c}+\alpha^{2}\right), \\
& \left\langle 0\left|\hat{d}_{\omega}^{\prime} \hat{d}_{\omega^{\prime}}^{\prime}\right| 0\right\rangle=g(\omega) g\left(\omega^{\prime}\right) \sinh r_{\omega} \sinh r_{\omega^{\prime}}\left(E_{d d}+\alpha^{* 2}\right) \text {, } \\
& \left\langle 0\left|\hat{c}_{\omega}^{\prime} \hat{d}_{\omega^{\prime}}^{\prime}\right| 0\right\rangle=g^{*}(\omega) g\left(\omega^{\prime}\right) \cosh r_{\omega} \sinh r_{\omega^{\prime}}\left(E_{c d}-|\alpha|^{2}\right), \\
& \left\langle 0\left|\hat{c}_{\omega}^{\prime \dagger} \hat{d}_{\omega^{\prime}}^{\prime}\right| 0\right\rangle=g(\omega) g\left(\omega^{\prime}\right) \cosh r_{\omega} \sinh r_{\omega^{\prime}}\left(\bar{E}_{c d}-\alpha^{* 2}\right) \text {, }
\end{aligned}
$$

where

$$
\begin{aligned}
E_{c} & =\mathcal{I}_{s}(\cosh r-1)^{2}+\mathcal{I}_{c} \sinh ^{2} r, \\
E_{d} & =\mathcal{I}_{c}(\cosh r-1)^{2}+\mathcal{I}_{s} \sinh ^{2} r, \\
E_{c c} & =\sinh r\left[\left(\mathcal{I}_{c}+\mathcal{I}_{s}\right)(\cosh r-1)+1\right], \\
E_{d d} & =\sinh r\left[\left(\mathcal{I}_{c}+\mathcal{I}_{s}\right)(\cosh r-1)-1\right], \\
E_{c d} & =-\cosh r(\cosh r-1)\left(\mathcal{I}_{c}+\mathcal{I}_{s}\right), \\
\bar{E}_{c d} & =-\sinh r(\cosh r-1)\left(\mathcal{I}_{c}+\mathcal{I}_{s}\right) .
\end{aligned}
$$

Other vacuum expectation values are either zero or complex conjugates of the ones above. From Eqs. (9) and (10), the vacuum expectation value of the total Minkowski particle number is 


$$
\langle 0|\hat{N}| 0\rangle=|\alpha|^{2}\left(\mathcal{I}_{c}+\mathcal{I}_{s}\right)+\left(\mathcal{I}_{c} E_{c}+\mathcal{I}_{s} E_{d}\right),
$$

and the variance of the total Minkowski particle number is

$$
\begin{aligned}
(\Delta N)^{2}= & \left\langle 0\left|\hat{N}^{2}\right| 0\right\rangle-\langle 0|\hat{N}| 0\rangle^{2} \\
= & |\alpha|^{2}\left[\left(\mathcal{I}_{c}+\mathcal{I}_{s}\right)+2\left(\mathcal{I}_{c}^{2} E_{c}+\mathcal{I}_{s}^{2} E_{d}\right)\right. \\
& +2 \mathcal{I}_{c}^{2} E_{c c} \cos (2 \phi)+2 \mathcal{I}_{s}^{2} E_{d d} \cos (2 \phi) \\
& \left.-4 \mathcal{I}_{c} \mathcal{I}_{s} E_{c d}-4 \mathcal{I}_{c} \mathcal{I}_{s} \bar{E}_{c d} \cos (2 \phi)\right],
\end{aligned}
$$

where $\phi$ is the displacement phase. In the homodyne detection, normalizing the variance of the particle number using the strength of the local oscillator gives the variance of the quadrature amplitude [20]. Here, the strength of the local oscillator is $\sim|\alpha|^{2}\left(\mathcal{I}_{c}+\mathcal{I}_{s}\right)$, so the variance of the quadrature amplitude is

$$
\begin{aligned}
V(\phi)= & \frac{(\Delta N)^{2}}{|\alpha|^{2}\left(\mathcal{I}_{c}+\mathcal{I}_{s}\right)} \\
= & \cosh (2 r)+4 \mathcal{I}_{c}\left(\mathcal{I}_{c}-1\right)(\cosh 2 r-2 \cosh r+1) \\
& +2 \sinh r\left[\left(2 \mathcal{I}_{c}-1\right)^{2} \cosh r-4 \mathcal{I}_{c}\left(\mathcal{I}_{c}-1\right)\right] \cos (2 \phi) .
\end{aligned}
$$

\section{APPENDIX B: UNIFORMLY ACCELERATED TWO-MODE SQUEEZER}

For a massless scalar field, the left-moving and rightmoving Rindler modes are decoupled. We consider a uniformly accelerated two-mode squeezer in the right Rindler wedge that couples the left-moving and right-moving Rindler modes. Entanglement between the left-moving and right-moving Rindler modes might be created by the

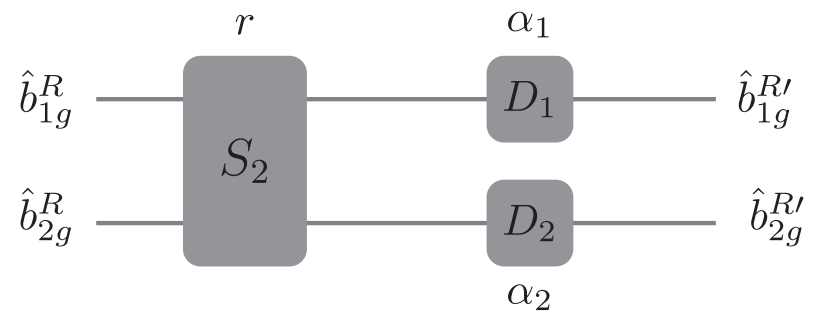

FIG. 7. A uniformly accelerated two-mode squeezer and the scheme of self-homodyne detection.

accelerated two-mode squeezer. One question of particular interest is, given that entanglement has been created as viewed by uniformly accelerated observers, whether entanglement between left-moving and right-moving fields exists as observed by inertial observers.

The unitary characterizing the uniformly accelerated two-mode squeezer is given by Eq. (17). Similar to the case of the uniformly accelerated single-mode squeezer, two uniformly accelerated displacements, $\hat{D}_{1}\left(\alpha_{1}\right)$ and $\hat{D}_{2}\left(\alpha_{2}\right)$, are introduced to realize the homodyne detection, where $\alpha_{1}=\left|\alpha_{1}\right| e^{i \phi_{1}}$ and $\alpha_{2}=\left|\alpha_{2}\right| e^{i \phi_{2}}$. The relevant circuit is shown in Fig. 7. The two local oscillators are used to detect the left-moving and right-moving fields, as well as the correlations between them. In this Appendix, we derive the covariance matrix for the output state of the uniformly accelerated two-mode squeezer.

The input-output relations for the circuit in Fig. 7 can be derived straightforwardly. By further using the relations between the Rindler operators and Unruh operators [25], we can derive the input-output relations for the Unruh operators:

$$
\begin{aligned}
\hat{c}_{1 \omega}^{\prime}= & \hat{c}_{1 \omega}+g^{*}(\omega) \cosh r_{\omega}\left[(\cosh r-1) \int \mathrm{d} \omega^{\prime} g\left(\omega^{\prime}\right)\left(\hat{c}_{1 \omega^{\prime}} \cosh r_{\omega^{\prime}}+\hat{d}_{1 \omega^{\prime}}^{\dagger} \sinh r_{\omega^{\prime}}\right)\right. \\
& \left.+\sinh r \int \mathrm{d} \omega^{\prime} g^{*}\left(\omega^{\prime}\right)\left(\hat{c}_{2 \omega^{\prime}}^{\dagger} \cosh r_{\omega^{\prime}}+\hat{d}_{2 \omega^{\prime}} \sinh r_{\omega^{\prime}}\right)+\alpha_{1}\right] \\
\hat{d}_{1 \omega}^{\prime}= & \hat{d}_{1 \omega}-g(\omega) \sinh r_{\omega}\left[(\cosh r-1) \int \mathrm{d} \omega^{\prime} g^{*}\left(\omega^{\prime}\right)\left(\hat{c}_{1 \omega^{\prime}}^{\dagger} \cosh r_{\omega^{\prime}}+\hat{d}_{1 \omega^{\prime}} \sinh r_{\omega^{\prime}}\right)\right. \\
& \left.+\sinh r \int \mathrm{d} \omega^{\prime} g\left(\omega^{\prime}\right)\left(\hat{c}_{2 \omega^{\prime}} \cosh r_{\omega^{\prime}}+\hat{d}_{2 \omega^{\prime}}^{\dagger} \sinh r_{\omega^{\prime}}\right)+\alpha_{1}^{*}\right] \\
\hat{c}_{2 \omega}^{\prime}= & \hat{c}_{2 \omega}+g^{*}(\omega) \cosh r_{\omega}\left[(\cosh r-1) \int \mathrm{d} \omega^{\prime} g\left(\omega^{\prime}\right)\left(\hat{c}_{2 \omega^{\prime}} \cosh r_{\omega^{\prime}}+\hat{d}_{2 \omega^{\prime}}^{\dagger} \sinh r_{\omega^{\prime}}\right)\right. \\
& \left.+\sinh r \int \mathrm{d} \omega^{\prime} g^{*}\left(\omega^{\prime}\right)\left(\hat{c}_{1 \omega^{\prime}}^{\dagger} \cosh r_{\omega^{\prime}}+\hat{d}_{1 \omega^{\prime}} \sinh r_{\omega^{\prime}}\right)+\alpha_{2}\right] \\
\hat{d}_{2 \omega}^{\prime}= & \hat{d}_{2 \omega}-g(\omega) \sinh r_{\omega}\left[(\cosh r-1) \int \mathrm{d} \omega^{\prime} g^{*}\left(\omega^{\prime}\right)\left(\hat{c}_{2 \omega^{\prime}}^{\dagger} \cosh r_{\omega^{\prime}}+\hat{d}_{2 \omega^{\prime}} \sinh r_{\omega^{\prime}}\right)\right. \\
& \left.+\sinh r \int \mathrm{d} \omega^{\prime} g\left(\omega^{\prime}\right)\left(\hat{c}_{1 \omega^{\prime}} \cosh r_{\omega^{\prime}}+\hat{d}_{1 \omega^{\prime}}^{\dagger} \sinh r_{\omega^{\prime}}\right)+\alpha_{2}^{*}\right]
\end{aligned}
$$


It is then straightforward to calculate the vacuum expectation values of the products of two output Unruh operators. For the left-moving operators, we have

$$
\begin{aligned}
\left\langle 0\left|\hat{c}_{1 \omega}^{\dagger} \hat{c}_{1 \omega^{\prime}}^{\prime}\right| 0\right\rangle & =g(\omega) g^{*}\left(\omega^{\prime}\right) \cosh r_{\omega} \cosh r_{\omega^{\prime}}\left(E_{c}+\left|\alpha_{1}\right|^{2}\right), \\
\left\langle 0\left|\hat{d}_{1 \omega}^{\prime \dagger} \hat{d}_{1 \omega^{\prime}}^{\prime}\right| 0\right\rangle & =g^{*}(\omega) g\left(\omega^{\prime}\right) \sinh r_{\omega} \sinh r_{\omega^{\prime}}\left(E_{d}+\left|\alpha_{1}\right|^{2}\right), \\
\left\langle 0\left|\hat{c}_{1 \omega}^{\prime} \hat{c}_{1 \omega^{\prime}}^{\prime}\right| 0\right\rangle & =\alpha_{1}^{2} g^{*}(\omega) g^{*}\left(\omega^{\prime}\right) \cosh r_{\omega} \cosh r_{\omega^{\prime}}, \\
\left\langle 0\left|\hat{d}_{1 \omega}^{\prime} \hat{d}_{1 \omega^{\prime}}^{\prime}\right| 0\right\rangle & =\alpha_{1}^{* 2} g(\omega) g\left(\omega^{\prime}\right) \sinh r_{\omega} \sinh r_{\omega^{\prime}}, \\
\left\langle 0\left|\hat{c}_{1 \omega}^{\prime} \hat{d}_{1 \omega^{\prime}}^{\prime}\right| 0\right\rangle & =g^{*}(\omega) g\left(\omega^{\prime}\right) \cosh r_{\omega} \sinh r_{\omega^{\prime}}\left(E_{c d}-\left|\alpha_{1}\right|^{2}\right), \\
\left\langle 0\left|\hat{c}_{1 \omega}^{\prime \dagger} \hat{d}_{1 \omega^{\prime}}^{\prime}\right| 0\right\rangle & =-\alpha_{1}^{* 2} g(\omega) g\left(\omega^{\prime}\right) \cosh r_{\omega} \sinh r_{\omega^{\prime}} .
\end{aligned}
$$

For the right-moving operators, we have

$$
\begin{aligned}
\left\langle 0\left|\hat{c}_{2 \omega}^{\dagger} \hat{c}_{2 \omega^{\prime}}^{\prime}\right| 0\right\rangle & =g(\omega) g^{*}\left(\omega^{\prime}\right) \cosh r_{\omega} \cosh r_{\omega^{\prime}}\left(E_{c}+\left|\alpha_{2}\right|^{2}\right), \\
\left\langle 0\left|\hat{d}_{2 \omega}^{\prime \dagger} \hat{d}_{2 \omega^{\prime}}^{\prime}\right| 0\right\rangle & =g^{*}(\omega) g\left(\omega^{\prime}\right) \sinh r_{\omega} \sinh r_{\omega^{\prime}}\left(E_{d}+\left|\alpha_{2}\right|^{2}\right), \\
\left\langle 0\left|\hat{c}_{2 \omega}^{\prime} \hat{c}_{2 \omega^{\prime}}^{\prime}\right| 0\right\rangle & =\alpha_{2}^{2} g^{*}(\omega) g^{*}\left(\omega^{\prime}\right) \cosh r_{\omega} \cosh r_{\omega^{\prime}}, \\
\left\langle 0\left|\hat{d}_{2 \omega}^{\prime} \hat{d}_{2 \omega^{\prime}}^{\prime}\right| 0\right\rangle & =\alpha_{2}^{* 2} g(\omega) g\left(\omega^{\prime}\right) \sinh r_{\omega} \sinh r_{\omega^{\prime}}, \\
\left\langle 0\left|\hat{c}_{2 \omega}^{\prime} \hat{d}_{2 \omega^{\prime}}^{\prime}\right| 0\right\rangle & =g^{*}(\omega) g\left(\omega^{\prime}\right) \cosh r_{\omega} \sinh r_{\omega^{\prime}}\left(E_{c d}-\left|\alpha_{2}\right|^{2}\right), \\
\left\langle 0\left|\hat{c}_{2 \omega}^{\dagger} \hat{d}_{2 \omega^{\prime}}^{\prime}\right| 0\right\rangle & =-\alpha_{2}^{* 2} g(\omega) g\left(\omega^{\prime}\right) \cosh r_{\omega} \sinh r_{\omega^{\prime}} .
\end{aligned}
$$

For the products of the left-moving and right-moving operators, we have

$$
\begin{aligned}
& \left\langle 0\left|\hat{c}_{1 \omega}^{\prime \dagger} \hat{c}_{2 \omega^{\prime}}^{\prime}\right| 0\right\rangle=\alpha_{1}^{*} \alpha_{2} g(\omega) g^{*}\left(\omega^{\prime}\right) \cosh r_{\omega} \cosh r_{\omega^{\prime}}, \\
& \left\langle 0\left|\hat{d}_{1 \omega}^{\prime \dagger} \hat{d}_{2 \omega^{\prime}}^{\prime}\right| 0\right\rangle=\alpha_{1} \alpha_{2}^{*} g^{*}(\omega) g\left(\omega^{\prime}\right) \sinh r_{\omega} \sinh r_{\omega^{\prime}}, \\
& \left\langle 0\left|\hat{c}_{1 \omega}^{\prime} \hat{c}_{2 \omega^{\prime}}^{\prime}\right| 0\right\rangle=g^{*}(\omega) g^{*}\left(\omega^{\prime}\right) \cosh r_{\omega} \cosh r_{\omega^{\prime}}\left(E_{c c}+\alpha_{1} \alpha_{2}\right), \\
& \left\langle 0\left|\hat{d}_{1 \omega}^{\prime} \hat{d}_{2 \omega^{\prime}}^{\prime}\right| 0\right\rangle=g(\omega) g\left(\omega^{\prime}\right) \sinh r_{\omega} \sinh r_{\omega^{\prime}}\left(E_{d d}+\alpha_{1}^{*} \alpha_{2}^{*}\right), \\
& \left\langle 0\left|\hat{c}_{1 \omega}^{\prime} \hat{d}_{2 \omega^{\prime}}^{\prime}\right| 0\right\rangle=-\alpha_{1} \alpha_{2}^{*} g^{*}(\omega) g\left(\omega^{\prime}\right) \cosh r_{\omega} \sinh r_{\omega^{\prime}}, \\
& \left\langle 0\left|\hat{d}_{1 \omega}^{\prime} \hat{c}_{2 \omega^{\prime}}^{\prime}\right| 0\right\rangle=-\alpha_{1}^{*} \alpha_{2} g(\omega) g^{*}\left(\omega^{\prime}\right) \sinh r_{\omega} \cosh r_{\omega^{\prime}}, \\
& \left\langle 0\left|\hat{c}_{1 \omega}^{\prime \dagger} \hat{d}_{2 \omega^{\prime}}^{\prime}\right| 0\right\rangle=g(\omega) g\left(\omega^{\prime}\right) \cosh r_{\omega} \sinh r_{\omega^{\prime}}\left(\bar{E}_{c d}-\alpha_{1}^{*} \alpha_{2}^{*}\right), \\
& \left\langle 0\left|\hat{d}_{1 \omega}^{\prime \dagger} \hat{c}_{2 \omega^{\prime}}^{\prime}\right| 0\right\rangle=g^{*}(\omega) g^{*}\left(\omega^{\prime}\right) \sinh r_{\omega} \cosh r_{\omega^{\prime}}\left(\bar{E}_{c d}-\alpha_{1} \alpha_{2}\right) .
\end{aligned}
$$

The vacuum expectation values of the left-moving and right-moving Minkowski particle number are

$$
\begin{aligned}
& \left\langle 0\left|\hat{N}_{1}\right| 0\right\rangle=\left|\alpha_{1}\right|^{2}\left(\mathcal{I}_{c}+\mathcal{I}_{s}\right)+\left(\mathcal{I}_{c} E_{c}+\mathcal{I}_{s} E_{d}\right), \\
& \left\langle 0\left|\hat{N}_{2}\right| 0\right\rangle=\left|\alpha_{2}\right|^{2}\left(\mathcal{I}_{c}+\mathcal{I}_{s}\right)+\left(\mathcal{I}_{c} E_{c}+\mathcal{I}_{s} E_{d}\right) .
\end{aligned}
$$

To accomplish the homodyne detection, we also need to know the strength of the local oscillators in the absence of signal: $\left\langle 0\left|\hat{N}_{10}\right| 0\right\rangle=\left|\alpha_{1}\right|^{2}\left(\mathcal{I}_{c}+\mathcal{I}_{s}\right), \quad\left\langle 0\left|\hat{N}_{20}\right| 0\right\rangle=\left|\alpha_{2}\right|^{2}\left(\mathcal{I}_{c}+\mathcal{I}_{s}\right)$. According to Eq. (3), the expectation values of the leftmoving and right-moving quadrature amplitudes can be found as

$$
\left\langle 0\left|\hat{X}_{1}\left(\phi_{1}\right)\right| 0\right\rangle=\left\langle 0\left|\hat{X}_{2}\left(\phi_{2}\right)\right| 0\right\rangle=0
$$

in the limit of $\left|\alpha_{1}\right| \gg 1$ and $\left|\alpha_{2}\right| \gg 1$.

Using Eq. (B2) and keeping terms to second order of $\alpha_{1}$, we find

$$
\begin{aligned}
\left\langle 0\left|\hat{N}_{1}\left(\phi_{1}\right) \hat{N}_{1}\left(\phi_{1}^{\prime}\right)\right| 0\right\rangle-\left\langle 0\left|\hat{N}_{1}\left(\phi_{1}\right)\right| 0\right\rangle\left\langle 0\left|\hat{N}_{1}\left(\phi_{1}^{\prime}\right)\right| 0\right\rangle & \\
= & \alpha_{1}^{*} \alpha_{1}^{\prime} \mathcal{I}_{c}+\alpha_{1} \alpha_{1}^{*} \mathcal{I}_{s}+\left(\alpha_{1}^{*} \alpha_{1}^{\prime}+\alpha_{1} \alpha_{1}^{* *}\right) \\
& \times\left(\mathcal{I}_{c}^{2} E_{c}+\mathcal{I}_{s}^{2} E_{d}-2 \mathcal{I}_{c} \mathcal{I}_{s} E_{c d}\right) .
\end{aligned}
$$

When $\phi_{1}=\phi_{1}^{\prime}$, we obtain the variance of the left-moving quadrature amplitude,

$$
\begin{aligned}
V_{1}\left(\phi_{1}\right) & =\frac{\left\langle 0\left|\hat{N}_{1}^{2}\left(\phi_{1}\right)\right| 0\right\rangle-\left\langle 0\left|\hat{N}_{1}\left(\phi_{1}\right)\right| 0\right\rangle^{2}}{\left\langle 0\left|\hat{N}_{10}\left(\phi_{1}\right)\right| 0\right\rangle} \\
& =1+2\left(\mathcal{I}_{c}^{2} E_{c}+\mathcal{I}_{s}^{2} E_{d}-2 \mathcal{I}_{c} \mathcal{I}_{s} E_{c d}\right) /\left(\mathcal{I}_{c}+\mathcal{I}_{s}\right)
\end{aligned}
$$

We are also interested in the case where $\phi_{1}^{\prime}=\phi_{1}+\pi / 2$ :

$$
\left\langle 0\left|\hat{X}_{1}\left(\phi_{1}\right) \hat{X}_{1}\left(\phi_{1}+\pi / 2\right)\right| 0\right\rangle=\frac{\left\langle 0\left|\hat{N}_{1}\left(\phi_{1}\right) \hat{N}_{1}\left(\phi_{1}+\pi / 2\right)\right| 0\right\rangle-\left\langle 0\left|\hat{N}_{1}\left(\phi_{1}\right)\right| 0\right\rangle\left\langle 0\left|\hat{N}_{1}\left(\phi_{1}+\pi / 2\right)\right| 0\right\rangle}{\sqrt{\left\langle 0\left|\hat{N}_{10}\left(\phi_{1}\right)\right| 0\right\rangle\left\langle 0\left|\hat{N}_{10}\left(\phi_{1}+\pi / 2\right)\right| 0\right\rangle}}=\frac{i}{\mathcal{I}_{c}+\mathcal{I}_{s}} .
$$

According to the symmetry between the left-moving and right-moving modes, similar results can be obtained:

$$
V_{2}\left(\phi_{2}\right)=1+2\left(\mathcal{I}_{c}^{2} E_{c}+\mathcal{I}_{s}^{2} E_{d}-2 \mathcal{I}_{c} \mathcal{I}_{s} E_{c d}\right) /\left(\mathcal{I}_{c}+\mathcal{I}_{s}\right)
$$

and 


$$
\left\langle 0\left|\hat{X}_{2}\left(\phi_{2}\right) \hat{X}_{2}\left(\phi_{2}+\pi / 2\right)\right| 0\right\rangle=\frac{i}{\mathcal{I}_{c}+\mathcal{I}_{s}} .
$$

To first order of $\alpha_{1} \alpha_{2}$, we find

$$
\begin{array}{r}
\left\langle 0\left|\hat{N}_{1}\left(\phi_{1}\right) \hat{N}_{2}\left(\phi_{2}\right)\right| 0\right\rangle-\left\langle 0\left|\hat{N}_{1}\left(\phi_{1}\right)\right| 0\right\rangle\left\langle 0\left|\hat{N}_{2}\left(\phi_{2}\right)\right| 0\right\rangle \\
\quad=\left(\alpha_{1} \alpha_{2}+\alpha_{1}^{*} \alpha_{2}^{*}\right)\left(\mathcal{I}_{c}^{2} E_{c c}+\mathcal{I}_{s}^{2} E_{d d}-2 \mathcal{I}_{c} \mathcal{I}_{s} \bar{E}_{c d}\right) .
\end{array}
$$

The vacuum expectation value of the product of the leftmoving and right-moving quadrature amplitudes is

$$
\begin{aligned}
& \left\langle 0\left|\hat{X}_{1}\left(\phi_{1}\right) \hat{X}_{2}\left(\phi_{2}\right)\right| 0\right\rangle \\
& =\frac{\left\langle 0\left|\hat{N}_{1}\left(\phi_{1}\right) \hat{N}_{2}\left(\phi_{2}\right)\right| 0\right\rangle-\left\langle 0\left|\hat{N}_{1}\left(\phi_{1}\right)\right| 0\right\rangle\left\langle 0\left|\hat{N}_{2}\left(\phi_{2}\right)\right| 0\right\rangle}{\sqrt{\left\langle 0\left|\hat{N}_{10}\left(\phi_{1}\right)\right| 0\right\rangle\left\langle 0\left|\hat{N}_{20}\left(\phi_{2}\right)\right| 0\right\rangle}} \\
& =2\left(\mathcal{I}_{c}^{2} E_{c c}+\mathcal{I}_{s}^{2} E_{d d}-2 \mathcal{I}_{c} \mathcal{I}_{s} \bar{E}_{c d}\right) \cos \left(\phi_{1}+\phi_{2}\right) /\left(\mathcal{I}_{c}+\mathcal{I}_{s}\right) .
\end{aligned}
$$

For Gaussian states, the covariance matrix is a very important quantity to characterize the state. In the special case where the expectation values of the quadrature amplitudes are zero, which is the case that we are considering, the covariance matrix contains full information of the state. We formally define an operator vector

$$
\begin{aligned}
\hat{\mathbf{x}} & \equiv\left(\hat{x}_{1}, \hat{p}_{1}, \hat{x}_{2}, \hat{p}_{2}\right)^{\mathrm{T}} \\
& =\left(\hat{X}_{1}(0), \hat{X}_{1}(\pi / 2), \hat{X}_{2}(0), \hat{X}_{2}(\pi / 2)\right)^{\mathrm{T}} .
\end{aligned}
$$

The covariance matrix $\mathbf{V}$ is defined as

$$
V_{i j}=\frac{1}{2}\left\langle\left\{\delta \hat{x}_{i}, \delta \hat{x}_{j}\right\}\right\rangle,
$$

where $\delta \hat{x}_{i}=\hat{x}_{i}-\left\langle\hat{x}_{i}\right\rangle$ and $\{$,$\} is the anticommutator. Using$ the fact that $\left\langle\hat{x}_{i}\right\rangle=0$ and Eqs. (B8)-(B13), we find that the nonvanishing components of the covariance matrix are

$$
\begin{aligned}
V_{11} & =V_{22}=V_{33}=V_{44} \\
& =1+2\left(\mathcal{I}_{c}^{2} E_{c}+\mathcal{I}_{s}^{2} E_{d}-2 \mathcal{I}_{c} \mathcal{I}_{s} E_{c d}\right) /\left(\mathcal{I}_{c}+\mathcal{I}_{s}\right), \\
V_{13} & =V_{31}=-V_{24}=-V_{42} \\
& =2\left(\mathcal{I}_{c}^{2} E_{c c}+\mathcal{I}_{s}^{2} E_{d d}-2 \mathcal{I}_{c} \mathcal{I}_{s} \bar{E}_{c d}\right) /\left(\mathcal{I}_{c}+\mathcal{I}_{s}\right) .
\end{aligned}
$$

By using Eq. (A4) and the relation $\mathcal{I}_{c}-\mathcal{I}_{s}=1$, we obtain the covariance matrix Eq. (18).

\section{APPENDIX C: AN ADDITIONAL DISPLACEMENT IN THE LEFT RINDLER WEDGE}

While, in general, it is not possible for the inertial detector to see a pure state if the squeezer and local oscillator are imposed on the right Rindler wedge, in this Appendix, we show that by appropriately adding an additional local oscillator in the left Rindler wedge, the inertial detector can see a pure state. Physically, this would require a mirror-image accelerated source to perform coordinated displacements of the quantum field in their reference frame.

In order to match the mode shape in the right Rindler wedge, the wave-packet mode in the left Rindler wedge is chosen as $g^{*}(\omega)$. The appearance of the complex conjugate comes from the fact that the coordinate time in the left Rindler wedge runs backward compared to that in the right Rindler wedge (and the Minkowski time coordinate). The displacement operator is thus $\hat{D}_{g^{*}}(\gamma)$, where $\gamma=|\gamma| e^{i \phi_{\gamma}}$ and $\phi_{\gamma}$ is the phase. We further require that the phase $\phi_{\gamma}$ satisfies $\phi_{\gamma}=-\phi$. For convenience, we define the ratio between the amplitude of the displacements in the left and right Rindler wedges as $z \equiv|\gamma| /|\alpha|$. From the general input-output relations of the Unruh modes, Eq. (6), we find

$$
\begin{aligned}
& \hat{c}_{\omega}^{\prime}=\hat{c}_{\omega}^{s}+\alpha g^{*}(\omega) L_{\omega}, \\
& \hat{d}_{\omega}^{\prime}=\hat{d}_{\omega}^{s}+\alpha^{*} g(\omega) M_{\omega},
\end{aligned}
$$

where $L_{\omega}=\cosh r_{\omega}-z \sinh r_{\omega}, M_{\omega}=z \cosh r_{\omega}-\sinh r_{\omega}$, and the operators $\hat{c}_{\omega}^{s}$ and $\hat{d}_{\omega}^{s}$ are the output Unruh operators in the absence of displacements. For a uniformly accelerated single-mode squeezer,

$\hat{c}_{\omega}^{s}=\hat{c}_{\omega}+g^{*}(\omega) \cosh r_{\omega}\left[\hat{b}_{g}^{R}(\cosh r-1)+\hat{b}_{g}^{R \dagger} \sinh r\right]$,

$\hat{d}_{\omega}^{s}=\hat{d}_{\omega}-g(\omega) \sinh r_{\omega}\left[\hat{b}_{g}^{R^{\dagger}}(\cosh r-1)+\hat{b}_{g}^{R} \sinh r\right]$.

The total Minkowski particle number operator

$\hat{N}=|\alpha|^{2} \int \mathrm{d} \omega|g(\omega)|^{2}\left(L_{\omega}^{2}+M_{\omega}^{2}\right)+\left(\hat{n}_{c}+\hat{n}_{d}\right)+\hat{Y}_{c}+\hat{Y}_{d}$,

where $\hat{n}_{c}$ and $\hat{n}_{d}$ are the output Unruh particle numbers in the absence of displacements,

$$
\hat{n}_{c}=\int \mathrm{d} \omega \hat{c}_{\omega}^{s \dagger} \hat{c}_{\omega}^{s}, \quad \hat{n}_{d}=\int \mathrm{d} \omega \hat{d}_{\omega}^{s \dagger} \hat{d}_{\omega}^{s} .
$$

Note that $\hat{Y}_{c}$ and $\hat{Y}_{d}$ are defined as

$$
\begin{aligned}
& \hat{Y}_{c}=\alpha^{*} \int \mathrm{d} \omega g(\omega) L_{\omega} \hat{c}_{\omega}^{s}+\alpha \int \mathrm{d} \omega g^{*}(\omega) L_{\omega} \hat{c}_{\omega}^{s \dagger}, \\
& \hat{Y}_{d}=\alpha \int \mathrm{d} \omega g^{*}(\omega) M_{\omega} \hat{d}_{\omega}^{s}+\alpha^{*} \int \mathrm{d} \omega g(\omega) M_{\omega} \hat{d}_{\omega}^{s \dagger} .
\end{aligned}
$$

To second order of $\alpha$, the variance of the total particle number is 


$$
(\Delta N)^{2}=\left\langle 0\left|\hat{Y}_{c}^{2}\right| 0\right\rangle+\left\langle 0\left|\hat{Y}_{d}^{2}\right| 0\right\rangle+2\left\langle 0\left|\hat{Y}_{c} \hat{Y}_{d}\right| 0\right\rangle
$$

For a uniformly accelerated single-mode squeezer, the expectation values $\left\langle 0\left|\hat{Y}_{c}^{2}\right| 0\right\rangle,\left\langle 0\left|\hat{Y}_{d}^{2}\right| 0\right\rangle$ and $\left\langle 0\left|\hat{Y}_{c} \hat{Y}_{d}\right| 0\right\rangle$ can be calculated straightforwardly from Eqs. (C5) and (C2):

$$
\begin{aligned}
\left\langle 0\left|\hat{Y}_{c}^{2}\right| 0\right\rangle & =|\alpha|^{2}\left(\mathcal{I}_{c}-2 z \mathcal{I}_{c s}+z^{2} \mathcal{I}_{s}\right)+2|\alpha|^{2}\left[E_{c}+E_{c c} \cos (2 \phi)\right]\left(\mathcal{I}_{c}-z \mathcal{I}_{s}\right)^{2}, \\
\left\langle 0\left|\hat{Y}_{d}^{2}\right| 0\right\rangle & =|\alpha|^{2}\left(z^{2} \mathcal{I}_{c}-2 z \mathcal{I}_{c s}+\mathcal{I}_{s}\right)+2|\alpha|^{2}\left[E_{d}+E_{d d} \cos (2 \phi)\right]\left(z \mathcal{I}_{c}-\mathcal{I}_{s}\right)^{2}, \\
\left\langle 0\left|\hat{Y}_{c} \hat{Y}_{d}\right| 0\right\rangle & =2|\alpha|^{2}\left[E_{c d}+\bar{E}_{c d} \cos (2 \phi)\right]\left(\mathcal{I}_{c}-z \mathcal{I}_{s}\right)\left(z \mathcal{I}_{c}-\mathcal{I}_{s}\right),
\end{aligned}
$$

where the new integral $\mathcal{I}_{c s}$ is defined as

$$
\mathcal{I}_{c s}=\int \mathrm{d} \omega|g(\omega)|^{2} \cosh r_{\omega} \sinh r_{\omega} .
$$

Therefore, the variance of the quadrature amplitude is

$$
\begin{aligned}
V(\phi)= & \frac{\left\langle 0\left|\hat{N}^{2}\right| 0\right\rangle-\langle 0|\hat{N}| 0\rangle^{2}}{\left\langle 0\left|\hat{N}_{0}\right| 0\right\rangle} \\
= & 1+2\left\{[ E _ { c c } ( \mathcal { I } _ { c } - z \mathcal { I } _ { s } ) ^ { 2 } + E _ { d d } ( z \mathcal { I } _ { c } - \mathcal { I } _ { s } ) ^ { 2 } + 2 \overline { E } _ { c d } ( \mathcal { I } _ { c } - z \mathcal { I } _ { s } ) ( z \mathcal { I } _ { c } - \mathcal { I } _ { s } ) ] \operatorname { c o s } ( 2 \phi ) \left[E_{c}\left(\mathcal{I}_{c}-z \mathcal{I}_{s}\right)^{2}+E_{d}\left(z \mathcal{I}_{c}-\mathcal{I}_{s}\right)^{2}\right.\right. \\
& \left.\left.+2 E_{c d}\left(\mathcal{I}_{c}-z \mathcal{I}_{s}\right)\left(z \mathcal{I}_{c}-\mathcal{I}_{s}\right)\right]\right\} /\left[\left(1+z^{2}\right)\left(\mathcal{I}_{c}+\mathcal{I}_{s}\right)-4 z \mathcal{I}_{c s}\right],
\end{aligned}
$$

where $\hat{N}_{0}$ can be obtained from Eq. (C3) by omitting $\hat{n}_{c}$ and $\hat{n}_{d}$. Notice that when $z=0$, we regain Eq. (13) as expected [see also Eq. (A6)].

In the narrow-bandwidth limit, namely, the central frequency $\omega_{0}$ of $g(\omega)$ is much greater than the bandwidth $\sigma$, we can approximate the integrals as

$$
\mathcal{I}_{c} \approx \cosh ^{2} r_{\omega_{0}}, \quad \mathcal{I}_{s} \approx \sinh ^{2} r_{\omega_{0}}, \quad \mathcal{I}_{c s} \approx \cosh r_{\omega_{0}} \sinh r_{\omega_{0}}
$$

By substituting these approximated integrals into Eq. (C9), it is straightforward to show that when

$$
z=\frac{2 \sqrt{\mathcal{I}_{c}\left(\mathcal{I}_{c}-1\right)}}{2 \mathcal{I}_{c}-1}
$$

the variance of the quadrature amplitude is

$$
V(\phi)=\cosh (2 r)+\sinh (2 r) \cos (2 \phi) .
$$

This implies $V_{\max } V_{\min }=1$, indicating that the detected state is pure.

More generally, it can be shown that for an arbitrary wave packet $g(\omega)$, there exists a nonzero $z$ such that $V_{\max } V_{\min }=1$. In fact, one can solve the equation $V_{\max } V_{\min }=1$ for $z$ based on the variance of the quadrature amplitude Eq. (C9). Therefore, by appropriately creating a particular additional local oscillator in the left Rindler wedge, but only by doing so, the inertial detector will see a pure state.
[1] W. G. Unruh, Notes on Black-Hole Evaporation, Phys. Rev. D 14, 870 (1976).

[2] P. C. W. Davies, Scalar Production in Schwarzschild and Rindler Metrics, J. Phys. A 8, 609 (1975).

[3] W. G. Unruh and R. M. Wald, What Happens When an Accelerating Observer Detects a Rindler Particle, Phys. Rev. D 29, 1047 (1984).

[4] T. D. Lee, Are Black Holes Black Bodies?, Nucl. Phys. B264, 437 (1986).

[5] S. Takagi, Vacuum Noise and Stress Induced by Uniform Acceleration-Hawking-Unruh Effect in Rindler Manifold of Arbitrary Dimension, Prog. Theor. Phys. Suppl. 88, 1 (1986).

[6] C. W. Misner, K. Thorne, and J. A. Wheeler, Gravitation (Freeman, San Francisco, 1973).

[7] S. W. Hawking, Particle Creation by Black Holes, Commun. Math. Phys. 43, 199 (1975).

[8] S. W. Hawking, Breakdown of Predictability in Gravitational Collapse, Phys. Rev. D 14, 2460 (1976).

[9] L. Susskind, L. Thorlacius, and J. Uglum, The Stretched Horizon and Black Hole Complementarity, Phys. Rev. D 48, 3743 (1993).

[10] C. R. Stephens, G. t'Hooft, and B. F. Whitings, Black Hole Evaporation without Information Loss, Classical Quantum Gravity 11, 621 (1994).

[11] S. D. Mathur, The Fuzzball Proposal for Black Holes: An Elementary Review, Fortschr. Phys. 53, 793 (2005).

[12] S. W. Hawking, M. J. Perry, and A. Strominger, Soft Hair on Black Holes, Phys. Rev. Lett. 116, 231301 (2016).

[13] V. Baccetti, R. B. Mann, and D. R. Terno, Role of Evaporation in Gravitational Collapse, Classical Quantum Gravity 35, 185005 (2018).

[14] A. Almheiri, D. Marolf, J. Polchinski, and J. Scully, Black Holes: Complementarity or Firewalls?, J. High Energy Phys. 02 (2013) 062. 
[15] S. L. Braunstein and S. Pirandola, Better Late than Never: Information Retrieval from Black Holes, Phys. Rev. Lett. 110, 101301 (2013).

[16] L. C. B. Crispino, A. Higuchi, and G. E. A. Matsas, The Unruh Effect and Its Applications, Rev. Mod. Phys. 80, 787 (2008).

[17] M. O. Scully and M.S. Zubairy, Quantum Optics (Cambridge University Press, Cambridge, England, 1997).

[18] A. I. Lvovsky and M. G. Raymer, Continuous-Variable Optical Quantum-State Tomography, Rev. Mod. Phys. 81, 299 (2009).

[19] C. Weedbrook, S. Pirandola, R. Garcia-Patron, N. J. Cerf, T. C. Ralph, J. H. Shapiro, and S. Lloyd, Gaussian Quantum Information, Rev. Mod. Phys. 84, 621 (2012).

[20] H.-A. Bachor and T. C. Ralph, A Guide to Experiments in Quantum Optics, 2nd ed. (Wiley-VCH, Weinheim, 2004).

[21] N. D. Birrell and P.C. W. Davies, Quantum Fields in Curved Space (Cambridge University Press, Cambridge, England, 1984).
[22] W. G. Unruh, Thermal Bath and Decoherence of Rindler Spacetimes, Phys. Rev. D 46, 3271 (1992).

[23] R. Parentani, The Recoils of the Accelerated Detector and the Decoherence of Its Fluxes, Nucl. Phys. B454, 227 (1995).

[24] N. Obadia and R. Parentani, Notes on Moving Mirrors, Phys. Rev. D 64, 044019 (2001).

[25] D. Su, C. T. M. Ho, R. B. Mann, and T. C. Ralph, Quantum Circuit Model for Non-inertial Objects: A Uniformly Accelerated Mirror, New J. Phys. 19, 063017 (2017).

[26] P. P. Rohde, W. Mauerer, and C. Silberhorn, Spectral Structure and Decompositions of Optical States, and Their Applications, New J. Phys. 9, 91 (2007).

[27] S. J. Olson and T.C. Ralph, Entanglement between the Future and Past in the Quantum Vacuum, Phys. Rev. Lett. 106, 110404 (2011).

[28] C. M. Wilson, G. Johansson, A. Pourkabirian, M. Simoen, J. R. Johansson, T. Duty, F. Nori, and P. Delsing, Observation of the Dynamical Casimir Effect in a Superconducting Circuit, Nature (London) 479, 376 (2011). 